\title{
Europeans Decide on Particle Strategy
}

\author{
The CERN Council approved a strategy update that prioritizes a $100-\mathrm{km}$ \\ circular collider, while still developing other options for future particle \\ physics projects.
}

By Michael Schirber

E uropean particle physicists have updated their strategy for the coming decades. Beyond current commitments, the community advocates pursuing a new facility at the CERN site outside Geneva-a circular collider with a circumference of 100 kilometers. Such a machine could serve a dual purpose: to act initially as a "Higgs factory" where electrons and positrons smash together at energies up to 350 $\mathrm{GeV}$, and to later scope out the high-energy frontier by colliding protons at up to $100-\mathrm{TeV}$ energies. The feasibility of this so-called Future Circular Collider (FCC) is still an open question, which is why the strategy also calls for continued research and development into accelerator technology, such as plasma acceleration and muon colliders.

Following a two-year-long process, the European Strategy for

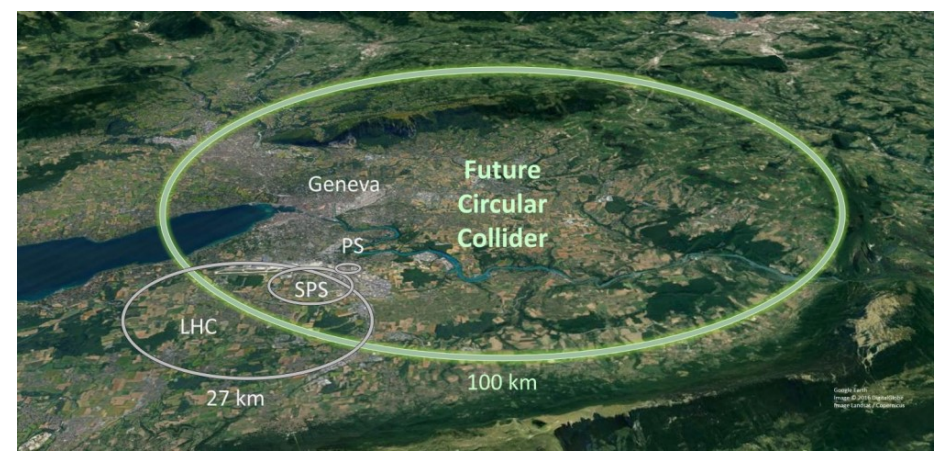

A map depicting where the 100-km-long Future Circular Collider could be built in relation to CERN's existing accelerator infrastructure.

Credit: CERN
Particle Physics Update was unanimously endorsed on June 19 by the CERN Council, which is the governing body of the CERN facility. The Update outlines a number of current and future priorities. In the near-term, the main initiatives for Europe are the high-luminosity upgrade of CERN's Large Hadron Collider (LHC) and continuing support of international neutrino experiments, such as the forthcoming Deep Underground Neutrino Experiment (DUNE) in the US. But beyond that, many questions remain. "CERN needs to have a project for after the LHC," says Halina Abramowicz, chair of the European Strategy Group, from Tel Aviv University in Israel.

The main objective of any post-LHC endeavor will be to look for new particles or phenomena that go beyond the standard model of particle physics. Physicists are still in the dark as to what this "new physics" will be, so the best way forward is to study the Higgs boson with greater precision, Abramowicz says. The Higgs is unique in that it should interact with all particles, even ones that physicists haven't detected yet. "The Higgs does not differentiate: if there is something out there, it will couple to it," Abramowicz explains.

Precision measurements of Higgs physics can be done with an electron-positron collider, but the exact design of such a Higgs factory is still undecided. The International Linear Collider (ILC) is one option, but the proposed host, Japan, has not yet committed to the project. Researchers at CERN have been developing the Compact Linear Collider (CLIC), which could potentially smash electrons and positrons at energies as high as $3 \mathrm{TeV}$. However, uncertainty about the energy where new physics might appear led the Strategy Group to decide on the FCC concept as the best option to pursue. The large ring-shaped 
tunnel could accommodate a Higgs factory and then later shift to colliding protons at energies 7 times greater than those of the LHC.

But pursuing the FCC won't be straightforward. "The FCC would be the machine that physicists most want," says Ursula Bassler, the president of the CERN Council. "However, we do not know if it's technically and financially feasible." Preliminary estimates suggest that such a collider would cost around 20 billion dollars, so involvement by countries outside of Europe will likely be necessary. "The scope and the science and technology challenges of such a Higgs factory would require a long-term global collaboration of the kind that the US is currently engaged in with the LHC and DUNE," says Fermilab's Marcela Carena, who was the US representative for the strategy's Physics Preparatory Group.

One possible wrinkle is that Chinese physicists have proposed the Circular Electron Positron Collider (CEPC), whose design is similar in size and scope to that of the FCC. "I think there is a competition between China and Europe," Bassler says. "However, there's also a lot of collaboration going on." As long as neither side has committed to a project, she thinks it can help spur innovation to have different groups working on the same research track.
Abramowicz stresses that the FCC is not the final word. By continuing research and development into accelerator technology, she believes particle physicists can remain flexible in the face of new developments in the scientific and political worlds. "From the input we received, it's clear that particle physicists are very excited about the FCC, but they do realize that it's not a given. So they want to make sure that we have alternatives."

Bassler is happy the process is complete. "In the beginning, every time I met a physicist at CERN cafeteria, I heard a different strategy." She feels the community has now converged on a common roadmap, in which the first step will be a thorough feasibility study of the FCC concept. At the same time, US particle physicists will be working on "Snowmass"-a community exercise led by the American Physical Society, which aims to draw up a particle physics vision for 2021. "The timing of the European Strategy Update fits well with the launch of the Snowmass process," Carena says.

Michael Schirber is a Corresponding Editor for Physics based in Lyon, France. 